\title{
MUTATIONS IN LUNG CANCER WITH CLINICAL SIGNIFICANCE
}

\author{
Patrick Pauwels \\ Department of Pathology, Antwerp University Hospital, Antwerp and Ghent University. \\ e-mail: patrick.pauwels@uza.be \\ doi:10.5152/tcb.2012.27
}

\begin{abstract}
Lung cancer is a complex and often fatal disease. The recent discoveries of activating mutations in EGFR and fusion genes involving ALK are milestones in a trend towards more personalized medicine in lung cancer. Targeting these alterations leads to survival benefit for our patients. EGFR and ALK testing can nowadays be considered as standard practice. Newer targets in the fight against lung cancer are discussed, such as MET and ROS.1 in adenocarcinoma. Also, new targets for squamous cell carcinoma are emerging, such as FGFR and DDR2.
\end{abstract}

Key words: Non small cell lung cancer, molecular testing-EGF(R), ALK, MET, FGFR, DDR2

\section{INTRODUCTION}

In the past years, tremendous efforts have been directed toward identifying potentially drugable molecular alterations in lung cancer. The sobering fact remains that, to date, we can identify therapeutic targets in only $20 \%$ of lung cancers (1).

The good news is that the clinical benefit from targeted therapies is greater than that for chemotherapy. In this review, we will focus on currently drugable mutations in non small cell lungs, and discuss some newer molecular targets.

\section{EPIDERMAL GROWTH FACTOR RECEPTOR (EGFR) MUTATION}

EGFR is part of the ErbB family of cell surface receptor tyrosine kinases, which control signal transduction pathways that regulate proliferation and apoptosis (2). These transmembrane receptors subsist as monomers on the cell surface. When these receptors bind to an extracellular signal (called "ligand"), they become activated. Known ligands for EGFR are epidermal growth factor "EGF), epiregulin and transform- ing growth factor alpha. A normal, resting EGFR is in a "blocked" state: it cannot dimerise because the dimerisation arms, located on the extracellular part of the receptor, are folded in such a way that they are not available on the surface of the molecule.

Ligand binding induces conformational changes; the extracellular part of the molecule unfolds and the dimerisation arms are now exposed, so that dimerisation can occur.

Homodimerisation means that EGFR couples with another EGFR. If another partner is involved (for example Her2 or Her3), then the process is called heterodimerisation. "Dimerisation" means "activation" of the receptor.

An active state means that a phosphate group from ATP can transfer to the cytoplasmic tail of the receptor, in particular to tyrosine residues in this tail ("tyrosine transphosphorylation"). Activation of the receptor means that by the process of dimerisation, the ATP binding pocket (cleft) of the receptor opens itself so that ATP can enter the receptor, allowing the transfer of phosphate by the kinase.

One way of blocking EGFR is to develop a small molecular molecule that competes with ATP in enter- 
ing the ATP binding pocket. In that way, the kinase activity of the receptor is blocked. This is the rationale for the development of gefitinib and erlotinib, so-called "tyrosine kinase inhibitors" (TKl's).

When treating unselected NSCLC patients with these TKI's, the results were disappointing. However, a subpopulation of patients experienced exquisite responses. In 2004, it was demonstrated that these responders had activating mutations localized within or near the ATP-binding site of the kinase $(3,4)$.

These activating EGFR mutants have a higher kinase activity than wild-type EGFR molecules. Recently, it has been shown that this is not due to an enhanced intrinsic catalytic potency of the mutant, but to a decreased threshold for dimerisation-mediated activation.

In other words: the mutant is predisposed to dimerize (5).

Why do NSCL tumors with activating EGFR mutations respond better to TKI's than WT EGFR tumors?

Erlotinib and gefitinib are in competition with ATP for entrance to the ATP binding pocket. These mutations alter the shape of the ATP binding pocket in such a way that TKI is the winner, and blocks the kinase activity. This mechanism also explains how resistant mutations can work: by changing the shape of the pocket in such a way that TKIs have more difficulty in entering the pocket.

In the end, all patients on TKI's develop resistance. Resistance to EGFR TKI's is due to a T790M mutation in $50 \%$ of cases.

In T790M, threonine is substituted by methionine in the catalytic cleft. This leads to a conformational change by which ATP is the winner in the competition between ATP and TKI (6).

\section{EGFR MUTATION TESTING}

Mutational status is the most important determinant of response to TKl's. Furthermore, patients with mutation-negative NSCLC have superior responses to conventional chemotherapy than to TKI administration, further indicating the importance of using the mutational status to guide the therapy section (7).

The American Society of Clinical Oncology published a provisional clinical opinion in which it is stated that "patients who are being considered for first-line therapy with an EGFR TKI should have their tumor tested for EGFR mutations to determine whether an EGFR TKI or chemotherapy is the appropriate first-line therapy.

They include patients with advanced NSCLC, and recommend testing mainly, but not exclusively, in patients with adenocarcinoma (8). In Europe, squamous cell carcinomas are excluded for testing, unless the patient is a non-smoker (9). Also, large-cell neuroendocrine carcinomas and mucinous adenocarcinomas are excluded.

The starting materials for EGFR mutation analysis are usually the material still available in the diagnostic samples. This EGFR analysis can be performed in materials from bronchoscopic biopsies, core biopsies from peripheral neoplasms and surgical resection specimens of the primary tumor. However, fine needle aspiration cytology or pleural effusion samples can also be used (by way of the cytology cell block technique) (10).

Is there enough tumor tissue on which to perform molecular testing? There is no definite answer to this question: how much is enough? The pathologist is best qualified to judge if a sample is adequate (considering the molecular testing technique used). Some studies have shown that EGFR mutations can be heterogeneously distributed in individual tumors.

A recent, well-documented study however, suggests that there are no differences in EGFR mutation status regardless of biopsy site (11).

There are several different methods of mutational analysis (12). In this regard, two important issues should be considered. First, some methods are capable of detecting any mutations present in the exons examined. Others, so-called allele-specific methods, are capable of detecting only certain types of mutation (e.g. length analysis for deletion) or the particular set of mutations the testing methodology is designed to detect. Those involved in testing should be aware of the mutations potentially found, or missed, by their chosen methodology. The second issue is that of test sensitivity, in this context referring to the level at which the test can detect mutated genome when diluted by wild-type genome. A test sensitivity of $5 \%$ suggests that a mutation could be detected to a dilution of $1 / 20$. Extracted tumor DNA will always include wild-type EGFR from non-neoplastic cells in the sample.

\section{ANAPLASTIC LYMPHOMA KINASE (ALK) REARRANGEMENT IN NSCLC}

ALK is also a tyrosine kinase receptor. Its ligand is unknown, at least in humans.

The enzyme was first found as a chimeric protein resulting from a chromosomal translocation, $\mathrm{t}(2 ; 5)$ (p23;q35) in anaplastic large cell lymphoma.

In 2007, a different ALK translocation was found in NSCLC (13). By an inversion, part of the ALK gene 
located on chromosome fused with part of another gene located on the same chromosome. This gene is the EML-4 gene (Echinoderm Microtubule-Associated protein-like 4 gene). What happens is that the tyrosine kinase-coding domain of the ALK gene fuses with part of the EML-4 gene, containing the code for the dimerisation domain. So, a new "fusion" protein is created, which contains a tyrosine kinase domain and a dimerisation domain. As soon as this new (fusion) gene is transcribed, dimerisation occurs and the new protein is active. A normal ALK receptor is located on the cell membrane and needs a ligand for activation. This new ALK fusion protein is already active in the cytoplasm, without the need for ligand binding.

The EML4-ALK fusion gene has been detected in about $3-5 \%$ of lung adenocarcinoma, and its transforming potential could be shown in NSCLC cell lines. As early as two years after the first description of this genetic aberration, results of a phase I/II clinical trial testing an oral c-met/ALK inhibitor (crizotinib) in relapsed NSCLC harboring EML4-ALK were presented at the ASCO annual meeting in 2009 reporting surprisingly high response rates. One year later, updated results were published with a confirmed partial response rate of $57 \%$ and a PFS rate of $72 \%$ after six months (14).

Based on the result of this and a further earlier phase trial, both with primary endpoint response rate $(\mathrm{RR})$, crizotinib was approved by the FDA for treatment of ALK-positive advanced NSCLC.

Current phase III trials are comparing crizotinib and chemotherapy in the first line and relapsed situation in ALK-positive patients. The remarkably short time period from the generic aberration to the approval of the drug (four years) emphasizes the current dynamics in the clinical development of personalized treatment approaches.

Three methods can detect ALK rearrangements: fluorescence in situ hybridization (FISH), reverse transcriptase-PCR (RT-PCR; multiplex RT-PCR) and immunohistochemistry (for aberrant expression of ALK protein) (12).

Multiplex RT-PCR may not be able to detect all the translocations involving the ALK gene. Indeed, not only EML-4 can act as a partner for ALK; other partners have been described and the list is growing.

The problem with immunohistochemistry-until nowis sensitivity. ALK-rearranged NSCLCs, irrespective of the fusion partner, invariably express ALK protein but at much lower levels than anaplastic large cell lymphomas. As a result, greater than $30 \%$ of ALK-rearranged NSCLCs are not identified by stand immunohisto- chemistry assays using the ALK antibody that has been used most prevalently worldwide (ALK 1, Dako USA, Carpentaria, California) $(15,16)$.

A novel antibody (D5F3, Cell Signaling Technology, Danvers, Massachusetts) seems to be a better alternative, but this has to be confirmed (16).

Up to now (despite some shortcomings), FISH is currently considered as the gold standard. FISH positivity for ALK rearrangement was (and is) required for entry into clinical trials.

\section{OTHER MOLECULAR ABNORMALITIES THAT SHOW PROMISE FOR TARGETED THERAPIES}

MET (mesenchymal-epithelial transition) gene copy number increases have been described and implicated in the pathogenesis of NSCL (17). Although true de novo MET amplification is rare in NSCLC, it is important as an acquired resistance mechanism in patients with activating EGFR mutations who progress on EGFR tyrosine kinase inhibitor (TKI). Up to $20 \%$ of patients on EGFR on TKI develop resistance by MET amplification. Crizotinib was in fact developed as a MET inhibitor.

Activity of crizotinib in patients with MET amplification have been demonstrated (16). An important fact in the MET story is also that $67 \%$ of adenocarcinomas, $60 \%$ of carcinoids, $57 \%$ of large cell carcinomas, $57 \%$ of squamous cell carcinomas and $25 \%$ of small cell lung cancers strongly express MET.

Several MET inhibitors are currently under investigation.

Another player in the field is ROS1. ROS1 gene rearrangement defines a molecular subset of NSCLS with distinct clinical characteristics that are similar to those observed in patients with ALK-rearranged NSCLC. Crizotinib shows in vitro activity and early evidence of clinical activity in ROS1 rearranged NSCLC (18).

All the advances described here, are more effective for patients with non-squamous NSCL. Recent data however, indicate that, similar to adenocarcinomas, squamous cell carcinomas (SCC) are also molecularly heterogeneous tumors.

Recent advances in basic science have also identified mutations, which are believed to be driver mutations. Two of them are discussed here.

The first one is fibroblastic growth factor 1 (FGFR 1) gene amplification. This FGFR 1 is one of the four members of the FGFR family. This receptor family has a prominent role in the regulation of cell proliferation and survival (19). Amplification of this gene has been seen in more than $20 \%$ of SCCs but only rarely in 
adenocarcinoma. A growing body of preclinical data shows that inhibition of FGFR signaling can result in anti-proliferative and/or pro-aptolic effects, both in vitro and in vivo. Several FGFR inhibitors are in development. Note that FGFR signaling deregulation also happens in other tumor types $(20,21)$.

Mutations in another kinase receptor, discoid domain receptor 2 (DDR2) have been described in about $4 \%$ of SCC (22). In cell line and xenograft models, growth of DDR2 mutants is inhibited by dasatinib, a tyrosine kinase inhibitor already in widespread use for the treatment of chronic myelogenous leukemia. Several phase II trials are ongoing.

\section{CONCLUSION}

Strenuous efforts are being made for eliciting a detailed analysis of the mutational spectrum of "drugable" oncogenes in NSCLC. Outside EGFR mutations and ALK rearrangements, it has become clear that other oncogenes such as MET, ROS, FGFR and DDR2 are important players in the field. It is foreseeable that in the near future most patients with NSCLC will have their specific therapy delineated by tumor genotyping.

\section{REFERENCES}

1. PT Cagle, LR Chirieac. Advances in treatment of lung cancer with targeted therapy. Arch Pathol Lab Med 2012;136:504-9. [CrossRef]

2. Sharma SV, Bell DW, Settleman J, Haber DA. Epidermal growth factor mutations in lung cancer. Nat Rev Cancer 2007;7:169-81. [CrossRef]

3. Lynch TS, Bell DW, Sordella R, et al. Activating mutations in the EGFR underlying responsiveness of NSCLC to gefitinib. N Engl J Med 2004; 350:2129-39. [CrossRef]

4. Paez JG, Jänne PA, Lee JC, et al. EGFR mutations in lung cancer: correlation with clinical responses to gefitinib therapy. Science 2004;304:1497-500. [CrossRef]

5. Shan Y, Eastwood MP, Zhang X, et al. Oncogenic mutations counteract intrinsic disorder in the EGFR kinase and promote receptor dimerisation. Cell 2012;149:860-70. [CrossRef]

6. Pirker R, Herth FJ, Kerr KM, et al. Consensus for EGFR mutation testing in NSCLC: results from a European workshop. J Thorac Oncol 2010;5;1706-13. [CrossRef]

7. Mok TS, Wu YL, Thongprasert S, et al. Gefitinib or carboplatin-paclitaxel in pulmonary adenocarcinoma. $\mathrm{N}$ Engl $\mathrm{J}$ Med 2009;361:947-57. [CrossRef]
8. Keedy VL, Temin S, Somerfield MR, et al. ASCO provisional clinical opinion: EGFR mutation testing for patients with advances non-small cell lung cancer considering first line EGFR tyrosine kinase inhibitor therapy. J Clin Oncol 2011;29:2121-7. [CrossRef]

9. Pirker R, Herth FJ, Kerr KM. Consensus for EGFR mutation testing in NSCLC: results from a European workshop. J Thorac Oncol 2010;10:1706-13. [CrossRef]

10. Bozetti C, Negri FV, Azzoni C. EGFR and KRAS gene expression: reliability of mutation analysis on cytological samples. Diagn Cytopathol 26 JUL 2012 DOI.10.1002/dc. 22905.

11. Yatabe $\mathrm{Y}$, Matsuo $\mathrm{K}$, Mitsudomi T. Heterogeneous distribution of EGFR mutations is extremely rare in lung adenocarcinoma. J Clin Oncol 2011;29:2972-7. [CrossRef]

12. Kerr K. Personalized medicine for lung cancer. New Challenges for Pathology. Histopathology 2012;60:531-46. [CrossRef]

13. Soda M, Choi YL, Enomoto M, et al. Identification of the transforming EML4-ALK fusion gene in NSCLC. Nature 2007;448:561-6. [CrossRef]

14. Kwak EL, Bang YJ, Camidge DR, et al. Anaplastic lymphoma kinase inhibition in NSCL. N Engl J Med 2010;363: 1693-703. [CrossRef]

15. Mino-Kenudson M, Mark EJ. Reflux testing for EGFR mutation and ALK FISH in NSCLC. Arch Pathol Lab Med 2011;135:655-64.

16. Miro-Kenudson M, Chirieac LR, Law K, et al. A novel highly sensitive antibody allows for the routine detection of ALK-rearranged lung adenocarcinomas by standard immunohistochemistry. Clin Cancer Res 2010;16:1561-71. [CrossRef]

17. Bergethon $\mathrm{K}$, Shaw A, Ou SH, et al. ROS1 rearrangements define a unique molecular class of lung cancers. J Clin Oncol 2012;30:863-70. [CrossRef]

18. Kim ES, Salgia R. MET pathway as a therapeutic target. J Thorac Oncol 2009;4:444-7. [CrossRef]

19. Turner N, Grosse R. Fibroblastic growth factor signaling from development to cancer. Nat Rev Cancer 2010;10: 116-29. [CrossRef]

20. Weiss J, Sos ML, Seidel D, et al. Frequent and focal FGFR1 amplification associates with therapeutically tractable FGFR1 dependency ibn squamous cell lung cancer. Sci Transl Med 2010;2:62ra93. [CrossRef]

21. Brooks AN, Kilgour E, Smith PD, et al. Molecular pathways: fibroblastic growth factor signaling: a new therapeutic opportunity in cancer. Clin Cancer Res 2012;18:185562. [CrossRef]

22. Hammerman PS, Sos ML, Ramos AH, et al. Mutations in the DDR2 kinase gene identify a novel therapeutic target in squamous cell lung cancer. Cancer Discov 2011;1:78-89. [CrossRef] 